\section{Novel MRI technique for assessing potential live kidney donors}

A safe, single alternative to the several separate procedures conventionally used to evaluate live kidney donors has been found by researchers at Mansoura University in Egypt. The new technique combines MRI of the kidney, ureter and renal blood vessels, and utilizes the contrast agent gadodiamide, which is injected into a vein and quickly distributes itself throughout the kidney, making the image clearer. This way, the anatomy, vasculature and glomerular filtration rate can be assessed simultaneously in order to check that both of the donor's kidneys are functioning well.

Examining a sample of 50 donors using $\mathrm{MRI}$, and comparing the findings with those obtained after surgical removal of the kidneys, the researchers visualized the number of renal arteries and veins with $\geq 96 \%$ accuracy, correctly estimated renal size and shape, and successfully detected any abnormalities in the ureter and bladder. They also found no significant difference between the glomerular filtration rate estimated from MRI angiography and that from total $24 \mathrm{~h}$ creatinine clearance.

The technique does have one major drawback, however; renal stones cannot be detected by MRI alone, so it needs to be used in conjunction with ultrasonography.

Despite this additional step, El-Diasty et al. advocate MRI as being a more cost-effective examination than the traditional combination of catheter angiography and excretory urography and, unlike CT another potential single-evaluation method, it does not come with associated risks such as overexposure to ionizing radiation.

Rachael Williams

Original article El-Diasty TA et al. (2005) Magnetic resonance imaging as a sole method for the morphological and functional evaluation of live kidney donors. BJU Int 96: 111-116

\section{Monitoring local prostate cancer recurrence after radiation therapy}

Salvage radical prostatectomy is indicated in a proportion of patients who fail externalbeam radiation therapy for prostate cancer, but the detection of local recurrence remains a challenge. A new report by Pucar et al. suggests that a combination of MRI and magnetic resonance spectroscopy (MRS) might be useful in this setting.

This retrospective evaluation included nine patients with a mean interval of 55 months between external-beam radiation therapy and MRI/MRS. Sextant biopsy, digital rectal examination, MRI and MRS were used to detect local recurrence, and all patients subsequently underwent salvage radical prostatectomy.

The investigators used step-section pathologic findings as the reference standard to calculate the sensitivity of MRI (68\%), MRS (77\%), biopsy (48\%), and digital rectal examination (16\%) for the detection of local recurrence. The estimated specificity of MRS $(78 \%)$ was lower than that of the other three techniques (>90\%).

These results suggest that the use of MRI and MRS might improve the sensitivity of sextant localization of prostate cancer recurrence. The authors suggest that these techniques might be incorporated into the routine assessment of patients exhibiting a rise in prostate-specific antigen after radiation therapy.

Ruth Kirby

Original article Pucar D et al. (2005) Prostate cancer: correlation of MR imaging and MR spectroscopy with pathologic findings after radiation therapy—initial experience. Radiology, 236: 545-553

\section{No increased risk of bladder cancer after prostate cancer radiation therapy}

A recent retrospective review of medical records from the Mayo Clinic Cancer Registry suggests that there is no increased risk of bladder cancer following external-beam radiation therapy (EBRT) for prostate cancer.

Chrouser and colleagues looked at the records of 1,743 patients who had received EBRT for prostate cancer at their clinic between 1980 and 1998, and identified those who had been subsequently diagnosed with bladder cancer. In total, there were over 12,353 man-years of postradiation follow-up in the patient records for the researchers to analyze, with the average follow-up period per patient being 7.1 years. 